\title{
Polarization and decoherence in a two-component Bose-Einstein Condensate
}

\author{
Le-Man Kuang ${ }^{1,2}$, Jin-Hui $\mathrm{Li}^{1}$ and Bambi $\mathrm{Hu}^{2,3}$ \\ ${ }^{1}$ Department of Physics, Hunan Normal University, Changsha 410081, China \\ ${ }^{2}$ Department of Physics, University of Houston, Houston, Texas 77204 \\ ${ }^{3}$ Department of Physics and Centre for Nonlinear Studies, \\ Hong Kong Baptist University, Hong Kong, China
}

\begin{abstract}
We theoretically investigate polarization properties of a two-component Bose-Einstein condensate (BEC) and influence of decoherence induced by environment on BEC polarization through introducing four BEC Stokes operators which are quantum analog of the classical Stokes parameters for a light field. BEC polarization states can be geometrically described by a Poincaré sphere defined by expectation values of BEC Stokes operators. Without decoherence, it is shown that nonlinear inter-atomic interactions in the BEC induce periodic polarization oscillations whose periods depend on the difference between self-interaction in each component and inter-component interaction strengths. In particular, when inter-atomic nonlinear self-interaction in each BEC component equals inter-component nonlinear interaction, Stokes vector associated with Stokes operators precesses around a fixed axis in the dynamic evolution of the BEC. The value of the processing frequency is determined by the strength of the linear coupling between two components of the BEC. When decoherence is involved, we find each component of the Stokes vector decays which implies that decoherence depolarizes the BEC.
\end{abstract}

PACS numbers: 05.30.-d, 03.75.Fi,42.25.Ja

\section{INTRODUCTION}

The experimental realization of Bose-Einstein condensation [1] and an atom laser 2] have sparked many theoretical and experimental studies of coherent atomic matter. It is well understood that matter-wave analog of nonlinear optical interactions is provided by inter-atomic collisions. In particular, in the $s$-wave scattering regime, two-body collisions are mathematically equivalent to a optical Kerr interaction. Hence, many of the concepts of first developed in optics can readily be extended to Bose-Einstein condensates (BEC). Recently, much attention has been paid to these lines, including the study of matter-wave solitons [3], phase conjugation [4], four-wave mixing [5], an atom laser [6], atom holography [7], etc. Especially, pulsed and continuous-wave atom lasers [8] and the four-wave mixing with matter waves [9] have been demonstrated experimentally. The experimental verification of the other predictions is also underway.

As is well known that polarization [10] is an important property that is common to all types of vector waves. Light waves possess this property and so do elastic and spin waves in solids, for example. In this paper we study BEC polarization that arises for matter waves by considering the two components of a two-component BEC as two polarizations. The motivation for present study is twofold. First, the BEC polarization is interesting in itself and deserves further examination. From a basic point of view, it significantly extends the already wellestablished range of analogies between light and matter waves. Second, more importantly perhaps, it may also have useful practical applications. We shall investigate nonlinear polarization dynamics of the BEC and study influence of decoherence induced by noncondensate atoms.
To begin we consider a two-component BEC described by the second quantized Hamiltonian 11.

$$
\begin{aligned}
\hat{H}= & \hat{H}_{1}+\hat{H}_{2}+\hat{H}_{n l}+\hat{H}_{l}, \\
\hat{H}_{i}= & \int d \mathbf{x} \hat{\psi}_{i}^{\dagger}(\mathbf{x})\left[-\frac{\hbar^{2}}{2 m} \nabla^{2}+V_{i}(\mathbf{x})\right. \\
& \left.+U_{i} \hat{\psi}_{i}^{\dagger}(\mathbf{x}) \hat{\psi}_{i}(\mathbf{x})\right] \hat{\psi}_{i}(\mathbf{x}),(i=1,2), \\
\hat{H}_{n l}= & U_{12} \int d \mathbf{x} \hat{\psi}_{1}^{\dagger}(\mathbf{x}) \hat{\psi}_{2}^{\dagger}(\mathbf{x}) \hat{\psi}_{1}(\mathbf{x}) \hat{\psi}_{2}(\mathbf{x}), \\
\hat{H}_{l}= & \Lambda \int d \mathbf{x}\left[\hat{\psi}_{1}^{\dagger}(\mathbf{x}) \hat{\psi}_{2}(\mathbf{x})+\hat{\psi}_{1}(\mathbf{x}) \hat{\psi}_{2}^{\dagger}(\mathbf{x})\right] .
\end{aligned}
$$

Here $i=1,2, \hat{\psi}_{i}(\mathbf{x})$ and $\hat{\psi}_{i}^{\dagger}(\mathbf{x})$ are the atomic field operators which annihilate and create atoms at position x, respectively. $\hat{H}_{1}$ and $\hat{H}_{2}$ describe the evolution of each component in the absence of intercommunion interactions. $\hat{H}_{n l}$ is a nonlinear interaction term, it describes inter-component collisions. $\hat{H}_{l}$ is a linear interaction term, it describes an atomic Josephson tunnelling process. $V_{i}(\mathbf{x})(i=1,2)$ are trapping potentials. Interactions between atoms are described by a nonlinear selfinteraction term $U_{i}=4 \pi \hbar^{2} a_{i}^{s c} / m$ and a term that corresponds the nonlinear interaction between different component $U_{12}=4 \pi \hbar^{2} a_{12}^{s c} / m$, where $a_{i}^{s c}$ is $s$-wave scattering lengths of component $i$ and $a_{12}^{s c}$ that between component 1 and 2. For simplicity, throughout this paper we set $\hbar=1$, and assume that $a_{1}^{s c}=a_{2}^{s c}=a^{s c}, V_{1}(\mathbf{x})=V_{2}(\mathbf{x})$.

For weak many-body interactions, i.e., for small number of condensate atoms, it has been well known that the Hamiltonian (1) can reduce to a two-mode Hamiltonian 11, 12

$$
\begin{aligned}
\hat{H}= & \omega_{0}\left(\hat{a}_{1}^{\dagger} \hat{a}_{1}+\hat{a}_{2}^{\dagger} \hat{a}_{2}\right)+q\left(\hat{a}_{1}^{\dagger 2} \hat{a}_{1}^{2}+\hat{a}_{2}^{\dagger 2} \hat{a}_{2}^{2}\right) \\
& +g\left(\hat{a}_{1}^{\dagger} \hat{a}_{2}+\hat{a}_{2}^{\dagger} \hat{a}_{1}\right)+2 \chi \hat{a}_{1}^{\dagger} \hat{a}_{1} \hat{a}_{2}^{\dagger} \hat{a}_{2},
\end{aligned}
$$


where $\hat{a}_{i}\left(\hat{a}_{i}^{+}\right)$is correspondent-mode annihilation (creation) operators with $\left[\hat{a}_{i}, \hat{a}_{i}^{\dagger}\right]=1$, and $q, \chi$ and $g$ are coupling constants which characterize the strength of nonlinear and linear interactions, respectively.

In general, the Hamiltonian (5) can not be exactly solved except the two limiting cases (i) $g=0, q \neq 0$ and/or $\chi \neq 0$; (ii) $g \neq 0$ and $q=\chi=0$. However, for the general case of $g \neq 0, q \neq 0$ and $\chi \neq 0$, an approximate analytic solution of the Hamiltonian (5) can be obtained by introducing a pair of bosonic operators: $\hat{A}_{1}=e^{-i g t}\left(\hat{a}_{1}+\hat{a}_{2}\right) / \sqrt{2}$ and $\hat{A}_{2}=i e^{i g t}\left(\hat{a}_{1}-\hat{a}_{2}\right) / \sqrt{2}$, which satisfy the usual bosonic commutation relation: $\left[\hat{A}_{i}, \hat{A}_{j}^{\dagger}\right]=\delta_{i j}$. Assume that the BEC is initially in the coherent state defined by

$\left|\alpha_{1}, \alpha_{2}\right\rangle=\exp \left[-\operatorname{frac12}\left(\left|\alpha_{1}\right|^{2}+\left|\alpha_{1}\right|^{2}\right)\right] \sum_{n, m=0}^{\infty} \frac{\alpha_{1}^{n} \alpha_{2}^{m}}{\sqrt{n ! m !}}|n, m\rangle$,

Where $\alpha_{1}$ and $\alpha_{2}$ are two arbitrary complex numbers. Under the rotating wave approximation, the energy and wave function of the system in the $\left(\hat{A}_{1}, \hat{A}_{2}\right)$ representation with the basis $\{\mid n, m)\}$ can be given by

$$
\begin{aligned}
E(n, m)= & \omega(n+m)+g(n-m)+\frac{1}{4}(3 q+2 \chi) \\
& \times(n+m)^{2}-\frac{q}{4}(n-m)^{2}-\chi n m, \\
|\Phi(t)\rangle= & e^{-\frac{1}{2} N} \sum_{n, m=0}^{\infty} \frac{1}{\sqrt{n ! m !}} u_{1}^{n}\left(i u_{2}\right)^{m} \\
& \left.\times e^{-i E(n, m) t} \mid n, m\right),
\end{aligned}
$$

where $n$ and $m$ take nonnegative integers, $\omega=\omega_{0}-(\chi-$ q) $/ 2, N=\left|\alpha_{1}\right|^{2}+\left|\alpha_{2}\right|^{2}=\left|u_{1}\right|^{2}+\left|u_{2}\right|^{2}$ is the total number of atoms with two new parameters defined by

$$
u_{1}=\frac{1}{\sqrt{2}}\left(\alpha_{1}+\alpha_{2}\right), \quad u_{2}=\frac{1}{\sqrt{2}}\left(\alpha_{1}-\alpha_{2}\right) .
$$

\section{BEC POLARIZATION STATES}

In order to discuss the polarization properties of the two-component BEC, it is convenient to define the Hermitian Stokes operators [10] as

$$
\begin{array}{ll}
\hat{S}_{0}=\left(\hat{a}_{1}^{+} \hat{a}_{1}+\hat{a}_{2}^{+} \hat{a}_{2}\right), & \hat{S}_{1}=\left(\hat{a}_{1}^{+} \hat{a}_{1}-\hat{a}_{2}^{+} \hat{a}_{2}\right), \\
\hat{S}_{2}=\left(\hat{a}_{1}^{+} \hat{a}_{2}+\hat{a}_{2}^{+} \hat{a}_{1}\right), & \hat{S}_{3}=i\left(\hat{a}_{2}^{+} \hat{a}_{1}-\hat{a}_{1}^{+} \hat{a}_{2}\right),
\end{array}
$$

which satisfy the commutation relations: $\left[\hat{S}_{j}, \hat{S}_{k}\right]=$ $2 i \epsilon_{j k l} \hat{S}_{l}$ and $\left[\hat{S}_{j}, \hat{S}_{0}\right]=0$. The noncommutability of the Stokes operators $S_{1}, S_{2}$, and $S_{3}$ precludes the simultaneous measurement of the physical quantities represented by them.

These Stokes operators are quantum analog of the classical Stokes parameters for a light field, i.e., $S_{\mu}=\left\langle\hat{S}_{\mu}\right\rangle$, where $\mu=0, \ldots, 3$. Any polarization state of the BEC

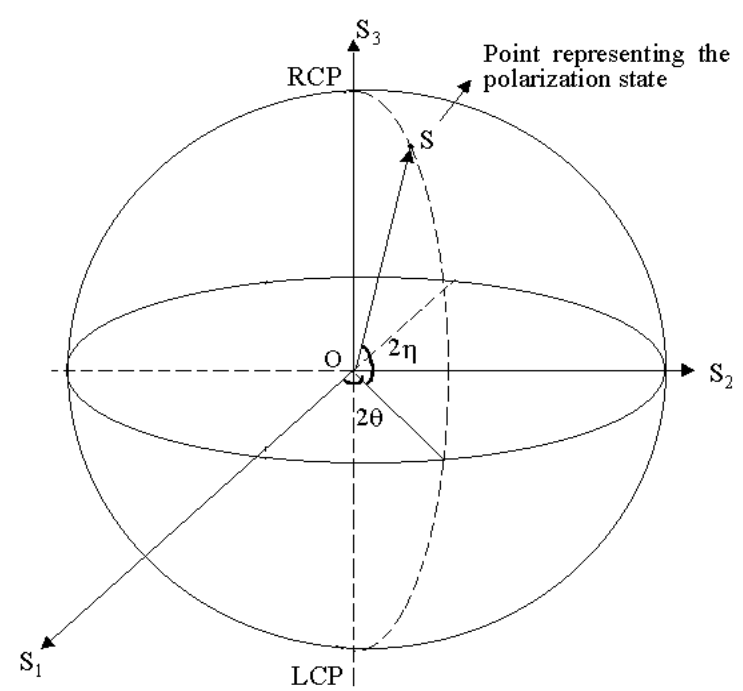

FIG. 1: Representation of the BEC polarization state on the Poincaré sphere.

can be completely described by the four Stokes parameters which can be written as a string: $\left\{S_{0}, S_{1}, S_{2}, S_{3}\right\}$. The first parameter expresses the total number of atoms in the BEC. The remaining three parameters describes the polarization state.

For a completely unpolarized BEC only the parameter $S_{0}$ is nonzero:

$$
S_{\mu}=S_{0}\{1 ; 0 ; 0 ; 0\} .
$$

The BEC is called completely polarized if the Stokes parameters satisfy the following relation:

$$
S_{0}=\left(S_{1}^{2}+S_{2}^{2}+S_{3}^{2}\right)^{1 / 2} .
$$

A partially polarized BEC is a mixture of a completely unpolarized BEC and a completely polarized BEC. All of the four Stokes parameters are independent, however, they satisfy the following inequality $S_{0}>\left(S_{1}^{2}+S_{2}^{2}+\right.$ $\left.S_{3}^{2}\right)^{1 / 2}$. And the degree of polarization can be measured by

$$
P=\frac{\sqrt{S_{1}^{2}+S_{2}^{2}+S_{3}^{2}}}{S_{0}} .
$$

For a partially polarized BEC $0<P<1$, a completely polarized BEC has $P=1$, while a completely unpolarized BEC has $P=0$.

BEC polarization states can be described by a Poincaré sphere plotted in Fig. 1. A particular polarization state of the BEC may be represented as a point in a 
three-dimensional Stokes space by introducing the Stokes vector $\mathbf{S}$ with Cartesian coordinates $S_{1}, S_{2}$ and $S_{3}$. The point lies on a Poincaré sphere 10 with radius $\left(S_{1}^{2}+S_{2}^{2}+S_{3}^{2}\right)^{1 / 2}$. If the Stokes parameters are known, the polarization azimuth $\Theta$ and the ellipticity angle $\eta$ defined by the following expressions, respectively,

$$
\begin{aligned}
\tan 2 \Theta & =\frac{S_{2}}{S_{1}}, \\
\sin 2 \eta & =\frac{S_{3}}{S_{0}} .
\end{aligned}
$$

If the total number of the atoms, and therefore $S_{0}$, the degree of polarization $P$, the polarization azimuth $\Theta$ and the ellipticity $\eta$ of the polarization ellipse are known, one may return the Stokes parameters $S_{1-3}$ :

$$
\begin{aligned}
& S_{1}=P S_{0} \cos (2 \eta) \cos (2 \Theta), \\
& S_{2}=P S_{0} \cos (2 \eta) \sin (2 \Theta), \\
& S_{3}=P S_{0} \sin (2 \eta) .
\end{aligned}
$$

If the BEC is totally polarized, the radius of the Poincaré sphere equals $S_{0}$. The 'north' and 'south' poles of the sphere correspond to right and left circular polarizations, respectively. Points on the 'equator' represent linear polarization with 'longitude' being twice the polarization azimuth $\Theta$. The 'latitude' of a point will give twice the angle of ellipticity $\eta$. Antipodes, i.e., points at opposite ends of a diameter of the sphere, are known as orthogonal polarizations. If the BEC is partially polarized the ratio between the Poincaré sphere radius and the Stokes parameter $S_{0}$ gives the degree of polarization (13).

We now examine the dynamical evolution of polarization of the BEC. From Eqs.(7) and (8) we can calculate the Stokes parameters. The results are

$$
\begin{aligned}
S_{0}= & N, \\
S_{1}= & 2\left|u_{1}\right|\left|u_{2}\right| \cos [4 g t+\theta(t)] \\
& \times \exp \left[-2 N \sin ^{2}\left(\frac{q-\chi}{2}\right) t\right], \\
S_{2}= & 2\left|\alpha_{1}\right|\left|\alpha_{2}\right| \cos \left(\varphi_{\alpha_{1}}-\varphi_{\alpha_{2}}\right), \\
S_{3}= & -2\left|u_{1}\right|\left|u_{2}\right| \sin [4 g t+\theta(t)] \\
& \times \exp \left[-2 N \sin ^{2}\left(\frac{q-\chi}{2}\right) t\right] .
\end{aligned}
$$

where we have set $u_{i}=\left|u_{i}\right| e^{i \varphi_{u_{i}}}$ and the symbol:

$$
\theta(t)=\left(\varphi_{u_{2}}-\varphi_{u_{1}}\right)+\left(\left|u_{2}\right|^{2}-\left|u_{1}\right|^{2}\right) \sin (q-\chi) t,
$$

Assume that the system is initially in a state $\mid \alpha_{1}, 0>$ of $\alpha_{1}$ being real and $\alpha_{2}=0$, which implies that the BEC is initially in a linear horizontal polarization state with the Stokes parameters given by $S_{\mu}=S_{0}\{1 ; 1 ; 0 ; 0\}$. From Eqs.(17) to (19), one can obtain some interesting polarization states of the BEC at some specific times. For instance, at times $t_{k}=(2 k+1) \pi /(8 g)$ with $k$ being an integer, the BEC is in a left-hand circular polarization state

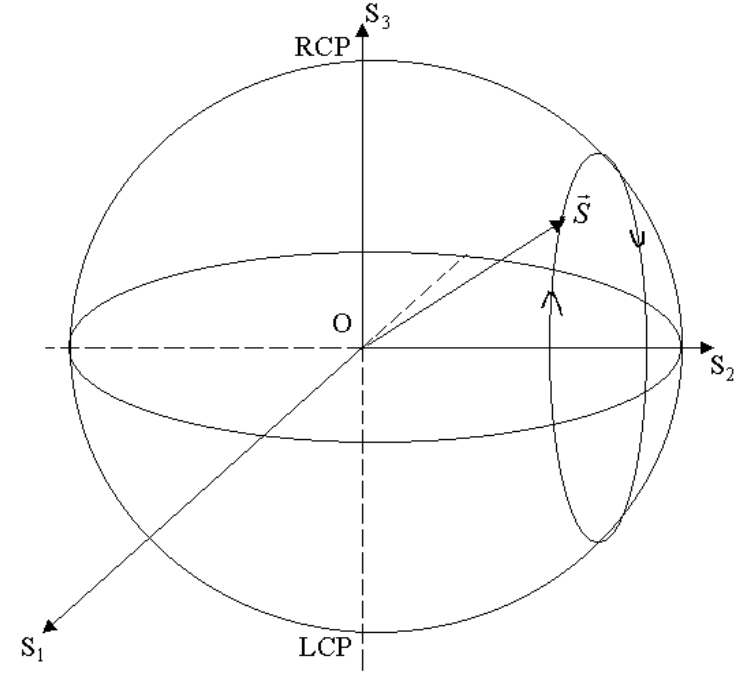

FIG. 2: Precession of the Stokes vector on the Poincaré sphere in the BEC evolution when $q=\chi$ and without decoherence.

with the Stokes parameters being $S_{\mu}=S_{0}\{1 ; 0 ; 0 ;-1\}$. When $t_{k}=(2 k-1) \pi /(8 g)$ with $k$ being an integer, the $\mathrm{BEC}$ is in a right-hand circular polarization state with the Stokes parameters being $S_{\mu}=S_{0}\{1 ; 0 ; 0 ; 1\}$. At times $t_{k}=k \pi /(2 g)$ with $k$ being an integer, the BEC returns the initial polarization state, a linear horizontal polarization state. When $t_{k}=(2 k+1) \pi /(4 g)$ with $k$ being an integer, one may find that the BEC is in a linearly vertical polarization state with the Stokes parameters given by $S_{\mu}=S_{0}\{1 ;-1 ; 0 ; 0\}$.

From Eqs. (11) to (14), we can obtain the degree of polarization

$$
\begin{aligned}
P(t)= & \frac{1}{N}\left\{\left(\left|u_{2}\right|^{2}-\left|u_{1}\right|^{2}\right)^{2}+4\left|u_{1}\right|^{2}\left|u_{2}\right|^{2}\right. \\
& \left.\times \exp \left[-4 N \sin ^{2}\left(\frac{q-\chi}{2}\right) t\right]\right\}^{1 / 2} .
\end{aligned}
$$

From above equation we see that: (i) the BEC system exhibits periodic polarization oscillations with a period $T=4 \pi /|q-\chi|$ which depends on the difference between the self-interaction and inter-component interaction. (ii) the degree of polarization depends only nonlinear interactions and the initial state of the BEC but not linear interaction due to the independence of $P(t)$ upon the linear coupling. (iii) When $q=\chi$, we obtain $P(t)=P(0)=1$, which means that the degree of polarization remains unchanged. It is interesting to note that when $q=\chi$, from Eqs. (17) to (19), we can find that the evolution equation 
of the Stokes vector takes the following form

$$
\frac{d \mathbf{S}}{d t}=-\mathbf{\Omega} \times \mathbf{S}
$$

where $\boldsymbol{\Omega}=4 g \hat{e}_{2}$ with $\hat{e}_{2}$ being the unit vector of the $S_{2}$ axis. Geometrically, Eq. (23) indicates that the Stokes vector $\mathbf{S}$ precesses around the $S_{2}$ axis in the dynamic evolution of the BEC. The value of the processing frequency $\Omega$ is determined by the strength of the BEC linear coupling. In Fig. 2 we plot the precession of the Stokes vector in the BEC evolution.

\section{INFLUENCE OF DECOHERENCE ON POLARIZATION}

We now consider the effect of the decoherence on the BEC polarization. As is well known, any system inevitably interacts with its environment. In fact, in current experiments on BECs of dilute alkali atomic gases condensate atoms continuously interact with noncondensate atoms (reservoir). Interaction between a BEC and environment causes decoherence [12, 13]. We use a reservoir consisting of an infinite set of harmonic oscillators to model environment of condensate atoms in a trap, and assume the total Hamiltonian [12] to be

$$
\begin{aligned}
\hat{H}_{T}= & \hat{H}+\sum_{k} \omega_{k} \hat{b}_{k}^{\dagger} \hat{b}_{k}+F(\{\hat{O}\}) \sum_{k} c_{k}\left(\hat{b}_{k}^{\dagger}+\hat{b}_{k}\right) \\
& +F(\{\hat{O}\})^{2} \sum_{k} \frac{c_{k}^{2}}{\omega_{k}^{2}},
\end{aligned}
$$

where the second term is the Hamiltonian of the reservoir. The last term in Eq.(24) is a renormalization term 14]. The third term in Eq.(24) represents the interaction between the system and the reservoir with a coupling constant $c_{k}$, where $\{\hat{O}\}$ is a set of linear operators of the system or their linear combinations in the same picture as that of $\hat{H}, F(\{\hat{O}\})$ is an operator function of $\{\hat{O}\}$. In order to enable what the interaction between the system and environment describes is decoherence not dissipation [15], we require that the linear operator $\hat{O}$ commutes with the Hamiltonian of the system $\hat{H}$. The concrete form of the function $F(\{\hat{O}\})$, which may be considered as an experimentally determined quantity. Therefore, the decohering interaction in (24) can not only describe decoherence caused by the effect of elastic collisions between condensate and non-condensate atoms, but also simulate decoherence caused by other decohering sources [16] through properly choosing the operator function of the system $F(\{\hat{O}\})$.

From Hamiltonian (24) through lengthy calculations we can get the Stokes parameters

$$
\begin{aligned}
S_{0}(t)= & N, \\
S_{1}(t)= & 2 \sum_{m, n} \sin ^{\prime} \phi_{m n}^{\prime}(t) \exp \left[-\gamma_{(m, n)(m+1, n-1)}(t)(26)\right. \\
S_{2}(t)= & \sum_{m, n} \cos ^{\prime \prime} \phi_{m n}^{\prime \prime}(t) \exp \left[-\gamma_{(m, n)(m, n)}(t)\right] \\
S_{3}(t)= & \sum_{m, n} \cos ^{\prime} \phi_{m n}^{\prime}(t) \exp \left[-\gamma_{(m, n)(m+1, n-1)}(t)\right] \\
& -\sum_{m, n} \sin ^{\prime \prime} \phi_{m n}^{\prime \prime}(t) \exp \left[-\gamma_{(m, n)(m, n)}(t)\right] .
\end{aligned}
$$

where we have introduced symbols

$$
\begin{aligned}
\sin ^{\prime} \phi_{m n}^{\prime}(t)= & \sqrt{n(m+1)}\left|\rho_{(m, n)(m+1, n-1)}(0)\right| \\
& \times \sin \left[2 g t+\phi_{(m, n)(m+1, n-1)}(t)\right] \\
\cos ^{\prime} \phi_{m n}^{\prime}(t)= & \sqrt{n(m+1)}\left|\rho_{(m, n)(m+1, n-1)}(0)\right| \\
& \times \cos \left[2 g t+\phi_{(m, n)(m+1, n-1)}(t)\right] \\
\sin ^{\prime \prime} \phi_{m n}^{\prime \prime}(t)= & (m-n)\left|\rho_{(m, n)(m, n)}(0)\right| \\
& \times \sin \left[2 g t+\phi_{(m, n)(m, n)}(t)\right] \\
\cos ^{\prime \prime} \phi_{m n}^{\prime \prime}(t)= & (m-n)\left|\rho_{(m, n)(m, n)}(0)\right| \\
& \times \cos \left[2 g t+\phi_{(m, n)(m, n)}(t)\right]
\end{aligned}
$$

where the damping factor and the phase shift induced by interaction between the BEC and reservoir are given by

$$
\begin{aligned}
\gamma_{\left(m^{\prime}, n^{\prime}\right)(m, n)}(t)= & v_{-}^{2}\left(m^{\prime}, n^{\prime} ; m, n\right) Q_{2}(t), \\
\phi_{\left(m^{\prime}, n^{\prime}\right)(m, n)}(t)= & v_{+}\left(m^{\prime}, n^{\prime} ; m, n\right) v_{-}\left(m^{\prime}, n^{\prime} ; m, n\right) Q_{1}(t) \\
& +\theta_{\left(m^{\prime}, n^{\prime}\right)(m, n)},
\end{aligned}
$$

Here we have introduced the notations

$$
\begin{aligned}
v_{ \pm}\left(n, m ; n^{\prime}, m^{\prime}\right)= & F(\{O(n, m)\}) \pm F\left(\left\{O\left(n^{\prime}, m^{\prime}\right)\right\}(335)\right. \\
\rho_{\left(m^{\prime}, n^{\prime}\right)(m, n)}(0)= & \mid \rho_{\left(m^{\prime}, n^{\prime}\right)(m, n)}(0) \\
& \times \mid \exp \left[-i \theta_{\left(m^{\prime}, n^{\prime}\right)(m, n)}\right]
\end{aligned}
$$

where the two reservoir-dependent functions are given by

$$
\begin{aligned}
Q_{1}(t)= & \int_{0}^{\infty} d \omega J(\omega) \frac{c^{2}(\omega)}{\omega^{2}} \sin (\omega t), \\
Q_{2}(t)= & 2 \int_{0}^{\infty} d \omega J(\omega) \frac{c^{2}(\omega)}{\omega^{2}} \\
& \times \sin ^{2}\left(\frac{\omega t}{2}\right) \operatorname{coth}\left(\frac{\beta \omega}{2}\right),
\end{aligned}
$$

where $J(\omega)$ is the spectral density of the reservoir, $c(\omega)$ is the continuum expression for $c_{k}$, and $\beta=1 / k_{B} T$ with $k_{B}$ and $T$ being the Boltzmann constant and temperature, respectively.

It is well known that decoherence corresponds to the decay of off-diagonal elements of the reduced density matrix of a quantum system. For the case under our consideration, the degree of decoherence is determined by 
the decaying factor given by Eq.(33) . It is interesting to note that if we choose a proper operator function $F(\{\hat{O}\})$ to make $F\left(\left\{O\left(m^{\prime}, n^{\prime}\right)\right\}\right)=F(\{O(m, n)\})$ for $\left(m^{\prime}, n^{\prime}\right) \neq(m, n)$, then we find that $\rho_{\left(m^{\prime}, n^{\prime}\right)(m, n)}(t)=$ $\rho_{\left(m^{\prime}, n^{\prime}\right)(m, n)}(0)$ which indicates that the decoherence-free evolution of the BEC is realized, and the BEC preserves its initial polarization state. From Eq. (26) into (28) we can immediately draw one important qualitative conclusion: since $Q_{2}(t)$ is positive, the existence of the decoherence is always to tend to make Stokes parameters decay. Therefore, decoherence always depolarizes the BEC.

From Eqs.(26) to (28), (33) and (34) we see that all necessary information about the effects of the reservoir on the polarization is contained in the spectral density of the reservoir. To proceed further let us now specialize to the Ohmic case [17] with the spectral distribution

$$
J(\omega)=\frac{\kappa \omega}{c^{2}(\omega)} \exp \left(-\frac{\omega}{\omega_{c}}\right),
$$

where $\omega_{c}$ is the high frequency cut-off, $\kappa$ is a positive characteristic parameter of the reservoir. With this choice, when $\omega_{c} t \gg 1$, at zero temperature we have $Q_{2}(t) \doteq \kappa \ln \left(\omega_{c} t\right)$, so that the components of the Stokes vector are given by

$$
\begin{aligned}
S_{1}(t)= & 2 \sum_{m, n} \sin ^{\prime} \phi_{m n}^{\prime}(t)\left(\omega_{c} t\right)^{-\kappa v_{-}^{2}(m, n ; m+1, n-1)},(40) \\
S_{2}(t)= & \sum_{m, n} \cos ^{\prime \prime} \phi_{m n}^{\prime \prime}(t)\left(\omega_{c} t\right)^{-\kappa v_{-}^{2}(m, n ; m, n)} \\
S_{3}(t)= & -\sum_{m, n} \sin ^{\prime \prime} \phi_{m n}^{\prime \prime}(t)\left(\omega_{c} t\right)^{-\kappa v_{-}^{2}(m, n ; m, n)} \\
& +\sum_{m, n} \cos ^{\prime} \phi_{m n}^{\prime}(t)\left(\omega_{c} t\right)^{-\kappa v_{-}^{2}(m, n ; m+1, n-1)}(\cdot 42)
\end{aligned}
$$

which indicate that Stokes parameters decay according to the 'power law'. Therefore, geometrically decoherence makes the Stokes vector contract towards the center of the Poincaré sphere according to the 'power law' with the time evolution.

At finite temperature, we have $Q_{2}(t) \doteq \kappa\left[\ln \left(\frac{\beta \omega_{c}}{2 \pi}\right)+\frac{\pi t}{\beta}\right]$, so that

$$
\begin{aligned}
S_{1}(t)= & 2 \sum_{m, n} \sin ^{\prime} \phi_{m n}^{\prime}(t)\left(\frac{\beta \omega_{c}}{2 \pi}\right)^{-\kappa v_{-}^{2}(m, n ; m+1, n-1)} \\
& \times \exp \left[-\kappa \pi \beta^{-1} v_{-}^{2}(m, n ; m+1, n-1) t\right],(43) \\
S_{2}(t)= & \sum_{m, n} \cos ^{\prime \prime} \phi_{m n}^{\prime \prime}(t)\left(\frac{\beta \omega_{c}}{2 \pi}\right)^{-\kappa v_{-}^{2}(m, n ; m, n)} \\
& \times \exp \left[-\kappa \pi \beta^{-1} v_{-}^{2}(m, n ; m, n) t\right] \\
S_{3}(t)= & \sum_{m, n} \cos ^{\prime} \phi_{m n}^{\prime}(t)\left(\frac{\beta \omega_{c}}{2 \pi}\right)^{-\kappa v_{-}^{2}(m, n ; m+1, n-1)} \\
& \times \exp \left[-\kappa \pi \beta^{-1} v_{-}^{2}(m, n ; m+1, n-1) t\right] \\
& -\sum_{m, n} \sin ^{\prime \prime} \phi_{m n}^{\prime \prime}(t)\left(\frac{\beta \omega_{c}}{2 \pi}\right)^{-\kappa v_{-}^{2}(m, n ; m, n)} \\
& \times \exp \left[-\kappa \pi \beta^{-1} v_{-}^{2}(m, n ; m, n) t\right]
\end{aligned}
$$

which indicate that at finite temperature Stokes parameter decay according to the 'exponential law'. Geometrically this means that decoherence makes the Stokes vector contracts toward the center of the Poincare sphere according to the 'exponential law' with the time evolution. Therefore, decoherence depolarizes the BEC.

From Eqs. (40) to (45) we see that a BEC initially in a completely polarized state $(P=1)$ evolves into a completely unpolarized state $(P=0)$ when the time approaches the infinity due to the influence of decoherence.

\section{CONCLUDING REMARKS}

In conclusion, we have examined the nonlinear polarization dynamics of a two-component BEC and influence of decoherence induced by environment on BEC polarization through introducing four BEC Stokes operators. We have shown that different polarization states of the BEC may be geometrically represented on a Poincaré sphere. Without decoherence, it has been found that nonlinear inter-atomic interactions in the BEC induce periodic polarization oscillations whose periods depend on the nonlinear interaction strengths. When nonlinear self-interaction $(q)$ equals inter-component nonlinear interaction $(\chi)$, the BEC initially in a completely polarized state remains a completely polarized state, however, the Stokes vector precesses around the $S_{2}$ axis with a precessing frequency determined by the linear coupling strength. When decoherence is taken into account, we find each component of the Stokes vector decays. This indicates that decoherence depolarizes the BEC. In particular, We have obtained analytical expressions of the Stokes parameters and found that for the reservoir-spectral density of the Ohmic case, the stokes parameters decay by the 'power law' at zero temperature, and the 'exponential law' at finite temperature, respectively.

\section{ACKNOWLEDGMENTS}

L.M.K. acknowledges support from the National "973" Research Plan, the National Natural Science Foundation, EYTF of the Educational Department of China, and Hunan Province STF. This work was also supported in part by grants from Hong Kong Research Grants Council (RGC) and the Hong Kong Baptist University Faculty Research Grant (FRG). 
[1] M.H. Anderson, J.R. Ensher, M.R. Matthews, C.E. Wieman, and E.A. Cornell, Science 269, 198 (1995); C.C. Bradley, C.A. Sackett, and R.G. Hulet, Phys. Rev. Lett. 75, 1687 (1995); K.B. Davis, M.-O. Mewes, M.R. Andrews, N.J. van Druten, D.S. Durfee, D.M. Kurn, and W. Ketterle, ibid. 75, 3969 (1995).

[2] M. R. Andrews, C. G. Townsend, H.-J. Miesner, D. S. Durfee, D. M. Kurn, and W. Ketterle, Science 275, 637 (1997); M.-O. Mewes, M. R. Andrews, D. M. Kurn, D. S. Durfee, C. G. Townsend, and W. Ketterle, Phys. Rev. lett. 78, 582(1997).

[3] S. A. Morgan, R. J. Ballagh, and K. Burnett, Phys. Rev. A 55, 4338 (1997); R. Dum, J. I. Cirac, M. Lewenstein, and P. Zoller, Phys. Rev. Lett. 80, 2972 (1998); P.D. Drummond and K.V. Kheruntsyan, ibid. 81, 3055 (1998).

[4] E.V. Goldstein and P. Meystre, Phys. Rev. A 59, 1509 (1999).

[5] M. Trippenbach, et al., Optics Express 3, 530 (1998); E.V. Goldstein and P. Meystre, Phys. Rev. A 59, 3896 (1999).

[6] H.M. Wiseman, Phys. Rev. A 56, 2068 (1997).

[7] O. Zobay, E. V. Goldstein, and P. Meystre, Phys. Rev. A 60, 3999 (1999).

[8] E. W. Hagley, L. Deng, M. Kozuma, J. Wen, K. Helmerson, S. L. Rolston, and W. D. Phillips, Science 283, 1706 (1999); S. Inouye, T. Pfau, S. Gupta, A. P. Chikkatur, A.
Görlitz, D. E. Pritchard, W. Ketterle, Nature 402, 641 (1999).

[9] L. Deng, E. W. Hagley, J. Wen, M. Trippenbach, Y. Band, P. S. Julienne, J. E. Simsarian, K. Helmerson, S. L. Rolston, W. D. Phillips, Nature 398, 218 (1999).

[10] E. Collett, Polarized Light: Fundamentals and Applications, (Marcel Dekker, Inc., New York, 1993).

[11] D. Gordon and C.M. Savage, Phys. Rev. A 59, 4623 (1999); J.I. Cirac, M. Lewenstein, K. Molmer, and P. Zoller, ibid. A 57, 1208 (1998); M.J. Steel and M.J. Collett, ibid. A 57,2920 (1998).

[12] L.-M. Kuang and Z.-W. Ouyang, Phys. Rev. A 61,023604 (2000).

[13] J. Anglin, Phys. Rev. lett. 79, 6 (1997); J. Ruostekoski and D. F. Walls, Phys. Rev. A 58, R50 (1998).

[14] A.O. Caldeira and A.J. Leggett, Ann. Phys. (N.Y.) 149, 374 (1983).

[15] C.P. Sun, H. Zhan, and X.F. Liu, Phys. Rev. A 58, 1810 (1998); L.-M. Kuang, X. Chen, G.-H. Chen, and M.-L. Ge, ibid. A 56, 3139 (1997).

[16] L.-M. Kuang, Hao-Sheng Zeng, and Zhao-Yang Tong, Phys. Rev. A 60, 3815 (1999).

[17] A.J. Leggett, S. Chakravarty, A.T. Dorsey, M.P.A. Fisher, A. Garg, and W. Zwerger, Rev.Mod. Phys. 59, 1 (1987). 